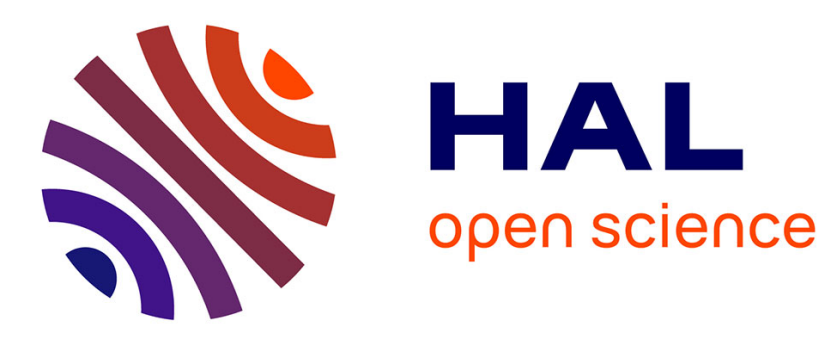

\title{
Effect of spanwise confinement on flag flutter: Experimental measurements
}

Olivier Doaré, David Mano, Juan Carlos Bilbao Ludena

\section{To cite this version:}

Olivier Doaré, David Mano, Juan Carlos Bilbao Ludena. Effect of spanwise confinement on flag flutter: Experimental measurements. Physics of Fluids, 2011, 23, pp.111704. 10.1063/1.3662127] . hal-01133649

\section{HAL Id: hal-01133649 \\ https://hal.science/hal-01133649}

Submitted on 20 Mar 2015

HAL is a multi-disciplinary open access archive for the deposit and dissemination of scientific research documents, whether they are published or not. The documents may come from teaching and research institutions in France or abroad, or from public or private research centers.
L'archive ouverte pluridisciplinaire HAL, est destinée au dépôt et à la diffusion de documents scientifiques de niveau recherche, publiés ou non, émanant des établissements d'enseignement et de recherche français ou étrangers, des laboratoires publics ou privés. 


\title{
Effect of spanwise confinement on flag flutter: Experimental measurements
}

\author{
Olivier Doaré, ${ }^{\text {a) }}$ David Mano, and Juan Carlos Bilbao Ludena \\ ENSTA-Paristech, Unité de Mécanique, 91761 Palaiseau, France
}

(Received 7 September 2011; accepted 15 October 2011; published online 29 November 2011)

\begin{abstract}
The effect of spanwise clearance on the critical velocity for fluttering of a cantilevered plate in a channel flow is addressed experimentally. It is found that the critical velocity is influenced by the presence of the walls when the ratio between the clearance and the length of the plate $\mathrm{C} / \mathrm{L}$ is less than 0.1 and slowly converges to the critical velocity predicted by models considering infinite span plates. These results are in good agreement with the predictions of a potential flow model taking into account spanwise confinement. (C) 2011 American Institute of Physics. [doi:10.1063/1.3662127]
\end{abstract}

The rest position of a cantilevered plate interacting with an axial flow becomes unstable at a critical value of the velocity. Oscillations of large amplitude are then observed. This phenomenon, referred to as flutter, is considered as a model for oscillatory instabilities encountered in many practical situations, such as snoring, ${ }^{1,2}$ fluid-elastic instabilities in nuclear engineering, ${ }^{3}$ panel flutter in aeronautics, ${ }^{4}$ or vibrations in the paper industry. ${ }^{5}$ Plate's fluttering has also recently been revisited in the context of energy harvesting of flows using induction ${ }^{6}$ or piezoelectricity. ${ }^{7,8}$

The 2D problem of a beam of finite length and infinite span in a potential flow has been first solved by Kornecki et al. ${ }^{4}$ Many other 2D models and numerical simulations followed. ${ }^{9}$ As these 2D works always underestimated the critical velocity when compared to experimental data, a 3D model for the flow was proposed by Eloy et al. ${ }^{10}$ Involving matching slender-body theory to $2 \mathrm{D}$ theory, this model evidenced the influence of the plate's aspect ratio on the critical velocity and was found to improve the flutter limit predictions. ${ }^{11}$ It was, however, admitted that it is possible to approach the limit predicted by $2 \mathrm{D}$ models by adding horizontal walls near both edges of the plate. ${ }^{1}$ A few experimental studies, ${ }^{1,2,12}$ although not focused on this particular effect, investigated such geometries. A reduction of the critical velocity was found but not important enough to reach the $2 \mathrm{D}$ limit, raising the question of the validity of the above mentioned assumption. This motivated the development of a 3D model taking into account the effect of spanwise boundaries, as proposed by Doaré et al. ${ }^{13}$ This model involves a matching between extended versions of the slender-body and 2D theories that take into account the spanwise confinement. The main result of the latter work is that the 2D limit is indeed reached when the gap tends to zero but with such a slow convergence that it should be almost impossible to attain this limit experimentally. Hence, it is now necessary to assess the validity of this improved 3D model with new experimental data. Even if the 2D limit is indeed impossible to reach, asymptotic behavior could be verified. This is the objective of the experimental work presented here. In the following, the 3D model of Eloy et al. ${ }^{10}$ will be referred to as the $3 \mathrm{D}_{\infty}$ model, as it considers a $3 \mathrm{D}$ unbounded flow. The

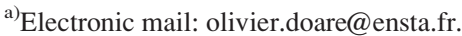

$3 \mathrm{D}$ model of Doaré et al. ${ }^{13}$ will be referred to as the $3 \mathrm{D}_{c}$ model, as it considers a 3D flow with a clearance $c$ between the plate and the wall.

The organisation of the letter is as follows: First, the experimental setup is described. Second, experimental results of critical velocities as functions of various parameters are presented. Finally, a discussion of the results is given.

The experimental setup is sketched on Fig. 1. It consists of a plexiglass rectangular wind tunnel of $10 \mathrm{~cm}$ width and $4 \mathrm{~cm}$ height connected upstream and downstream to a circular section of $9 \mathrm{~cm}$ internal radius through a smooth convergent and divergent. At the downstream end, a centrifugal fan regulated by a frequency inverter drives the flow, and the upstream circular section is connected to a convergent with a honeycomb screen. The height of the wind tunnel can be decreased by adding two additional plexiglass plates (see Fig. 1(c)). When smaller and more precise variations are desired, successive layers of adhesive tapes are added on both walls.

A mast is fixed in the wind tunnel and two steel plates are clamped in the mast. Mylar sheets are tightened between the steel plates so that the effective position of the clamped end of the flag under test is the downstream end of the steel plates. The Mylar sheets have a surface density of $0.21 \mathrm{~kg} \mathrm{~m}^{-2}$. The value of their flexural rigidity $D$ has been evaluated by measuring the first mode oscillation frequency of the flags in still air and has been found to be $D=8 \times 10^{-4} \mathrm{~m}^{2} \mathrm{~kg} \mathrm{~s}^{-2}$. It should be noted that although the Mylar sheets under study are formally plates, they will be often be referred to as "flags" due to their fluttering nature.

The flow velocity data of the present paper come from two sensors: a static-pitot tube and a hot-wire, placed at the exact inlet of the rectangular test section, as shown in Fig. 1. At this position, the channel is always of $4 \mathrm{~cm}$ height. Hence, when the flag is in a smaller channel (as in Fig. 1(c)), the velocity is estimated by assuming that the flow profile is constant and by invoking conservation of the flow rate. The unsteady deflection of the flag is measured with a laser displacement sensor (Keyence LK-G37) at an arbitrary position. Time series of the displacement is analysed in real time using LABVIEW software to compute the rms value of the plate's deflection.

The boundary layer thickness on the upper and lower walls of the channel has been quantified by performing flow 


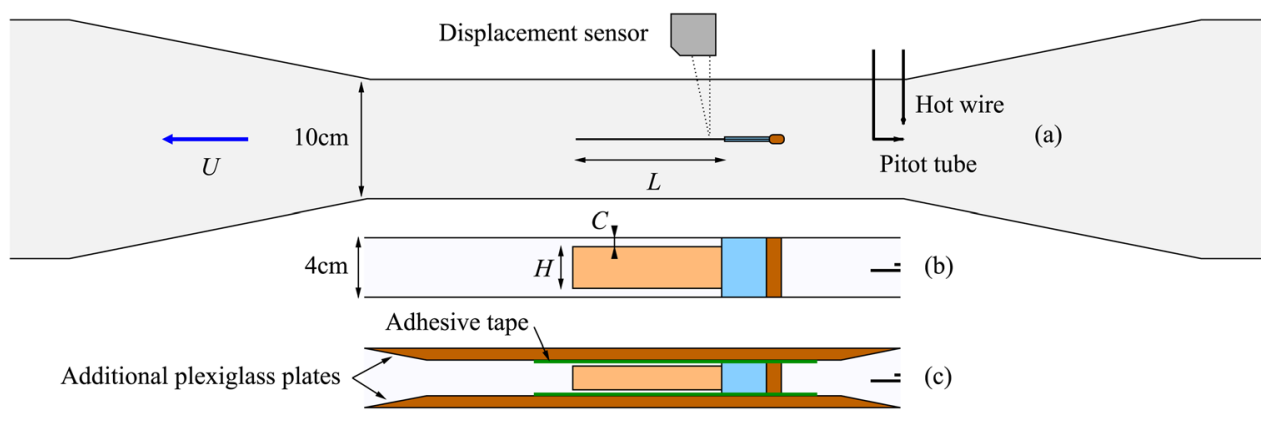

FIG. 1. (Color online) Sketch view of the experimental setup: (a) top view, (b) side view, (c) side view with additionnal plexiglass plates and adhesive tapes to decrease the channel width.

profile measurements at different values of the inlet flow velocity. At the pitot tube's location, the boundary layer is so small that it is not perceptible. The constant flow profile assumption is hence fully satisfied here. At a location near the flag's clamp, for the range of flow velocities of interest in the following, a boundary layer can be clearly evidenced. Its displacement length ${ }^{14}$ is found to be comprised between $\delta=0.7 \mathrm{~mm}$ and $\delta=1.2 \mathrm{~mm}$ and it scales as $U_{\infty}^{-1 / 2}$, indicating a Blasius-type boundary layer. ${ }^{14}$ Consequently, for the smallest channel heights considered in the present paper $(\sim 2 \mathrm{~cm})$, considering a constant velocity profile may induce an underestimation of approximately $10 \%$ on the velocity in the middle of the channel.

Let $R$ be the rms value of the unsteady deflection of the flag measured by the laser sensor. It is plotted as function of the flow velocity for two different sets of the geometrical parameters $H, L$, and $C$ on Fig. 2. These figures illustrate two typical behaviors of the flag when the flow velocity is varied. In both cases, at a value of the velocity below the critical velocity, random vibrations induced by unsteadiness of the flow around the plate are observed resulting in a value of $R$ around $4 \mathrm{~mm}$. In case (a), $R$ starts to increase at $U \sim 15 \mathrm{~m} / \mathrm{s}$ and slowly saturates to an approximate value of $6 \mathrm{~mm}$ when $U$ is further increased. The value of $R$ follows the same path when $U$ is decreased, suggesting a supercritical-type bifurcation. Conversely, case (b) displays a different behaviour $-R$ jumps abruplty at $U \sim 11 \mathrm{~m} / \mathrm{s}$ and saturates around $11 \mathrm{~mm}$. The path followed for decreasing velocities is different and restabilization occurs around $10.5 \mathrm{~m} / \mathrm{s}$. This hysteretic behaviour suggests a subcritical-type bifurcation. This discrepancy between different sets of parameters has already been observed by various authors, both numerically ${ }^{15-17}$ and experimentally. ${ }^{11}$ However, it was observed in the literature that even in the hysteretic case, the amplitude as function of the flow velocity is well fitted by a square root law, suggesting that the transition is supercritical. ${ }^{11}$ In the experiments reported here, the hysteresis was rarely perceptible and was at most of $10 \%$. It was, thus, decided to retain the increasing value of the critical velocity and plot the results with error bars of $10 \%$ in the following to take into account this hysteresis effect as well as uncertainities in the measurements and the underestimation of the velocity due to the boundary layer.

Before addressing further experiments, let us introduce the non-dimensional parameters that will be used. When dealing with an infinite span flag, only two parameters are necessary to fully characterize the problem, namely the mass-ratio and the non-dimensional flow velocity,

$$
M^{*}=\frac{\rho_{f} L}{M}, \quad U^{*}=\sqrt{\frac{M}{D}} L U,
$$

where $\rho_{f}$ is the fluid's density and $M$ is the plate's surface density. Finite width and spanwise confinement are taken into account through the aspect ratio ${ }^{10} h$ and the channel clearance ${ }^{13} c$, respectively. Their expressions are

$$
h=\frac{H}{L}, \quad c=\frac{C}{L} .
$$

A first set of experiments has been performed with flags of $8.2 \mathrm{~cm}$ length $\left(M^{*}=0.48\right)$ and different widths. In a channel of $2.2 \mathrm{~cm}$ height, for each of these flags, the critical velocity for apparition of flutter instability is measured. The resulting value of the non-dimensional critical velocity $U^{*}$ is plotted as function of $h$ on Fig. 3 and compared with the theoretical

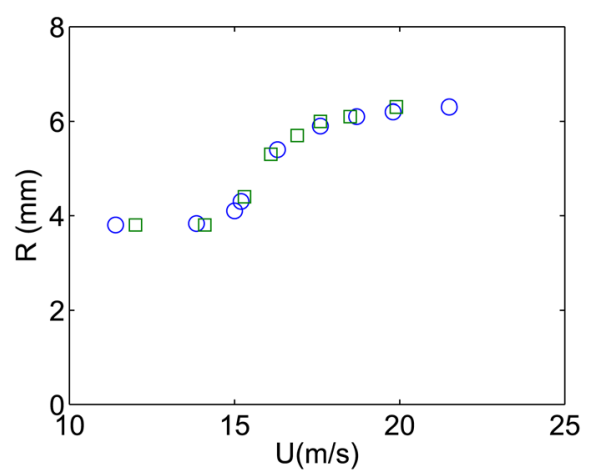

(a)

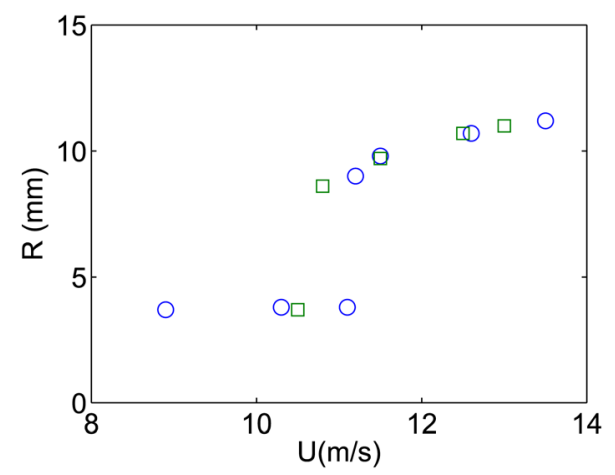

(b)

FIG. 2. (Color online) Typical evolution of the rms level of the displacement, measured at an arbitrary point near the clamped end of the plate; $(O)$ increasing velocity, ( $\square$ ) decreasing velocity, $L=86 \mathrm{~mm}$ (a), $H=15 \mathrm{~mm}$, $C=12 \mathrm{~mm}$; (b) $H=8 \mathrm{~mm}, C=16 \mathrm{~mm}$. 

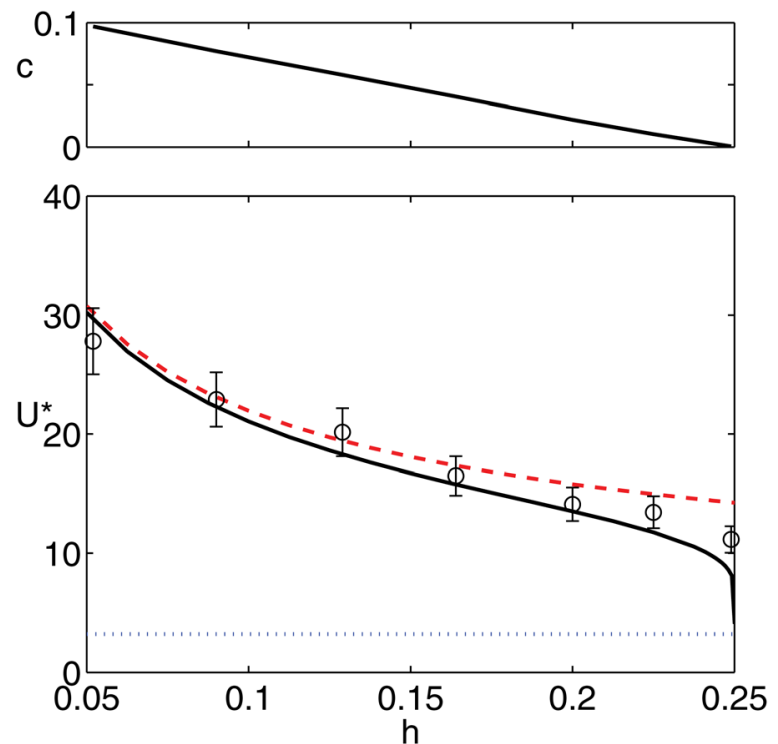

FIG. 3. (Color online) Evolution of the non-dimensional critical flow velocity for flutter instability $U^{*}$ as function of the aspect ratio $h$ of the plate; (o)

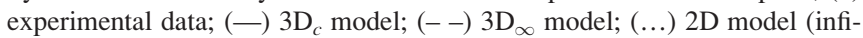
nite span). The experimental value of $c$ is plotted as reference above.

value predicted by the three models $\left(2 \mathrm{D}, 3 \mathrm{D}_{\infty}\right.$, and $\left.3 \mathrm{D}_{c}\right)$. For reference, the value of $c$ is plotted above as function of $h$. As emphasized in a previous article, the $3 \mathrm{D}_{c}$ model tends to the $3 \mathrm{D}_{\infty}$ model when $c$ is large, while it reaches the $2 \mathrm{D}$-model when $c \rightarrow 0$. The experimental data follows the 3D-models when $h$ is increased up to $\sim 0.15$. Above this value, the two 3D-models depart from each other, and the experimental data seem to lie in between the two. This scarcity of results does not permit to confirm the validity of the $3 \mathrm{D}_{c}$ model over the $3 \mathrm{D}_{\infty}$ model.

A second set of experiments is now presented. Here, only the gap $c$ is varied as opposed to the previous experiments. It is achieved by varying the channel height, while the flag geometry is kept constant. Three flags have been studied (Flag 1: $H=1.35 \mathrm{~cm}, L=8.2 \mathrm{~cm}$; Flag $2: H=1.65 \mathrm{~cm}, L=8.2 \mathrm{~cm}$; Flag 3: $H=3.9 \mathrm{~cm}, L=9.1 \mathrm{~cm}$ ). The resulting evolution of $U^{*}$ as function of $c$ is plotted in Fig. 4 and again compared with the three models. The behavior of the critical velocity as function of $c$ measured experimentally is correctly reproduced by the $3 \mathrm{D}_{c}$ model. A slight discrepancy is visible for $h=0.16$ and $h=0.2$ : the model seems to underestimate the critical velocity by $10 \%-15 \%$. In the inset of Fig. 4 , the difference between the critical velocity and that predicted by the $2 \mathrm{D}$ model is plotted as function of $c$. A power law $c^{0.15}$ is clearly evidenced for the $3 \mathrm{D}_{c}$ model, and the experimental data follow this asymptotic behavior.

In the experiments described above, $c=10^{-2}$ corresponds to $C \sim 1 \mathrm{~mm}$, which is the typical size of the displacement length of the boundary layer. Our experimental data exhibit a decrease of the critical velocity in the range $c \in\left[10^{-3}, 10^{-1}\right]$. This would suggest that the decreasing of the critical flow velocity, observed on Fig. 4 when $c$ is decreased, occurs when the flag's edges enter the boundary layer. One may then wonder if it is a coincidence or if the boundary layer can have such influence on the critical velocity. Three effects of the boundary layer may be distin-

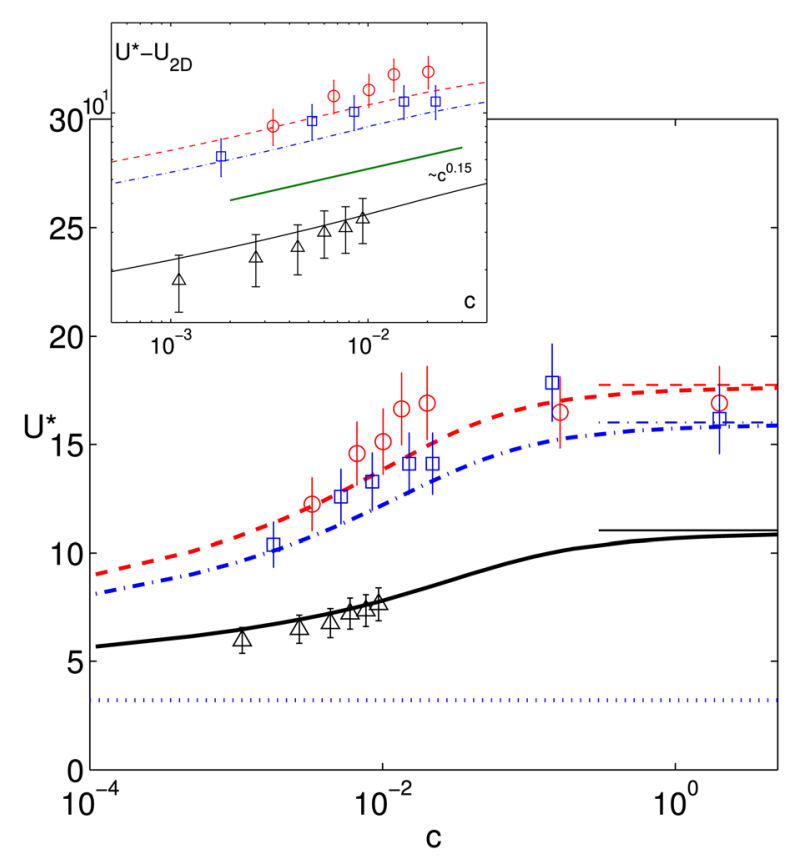

FIG. 4. (Color online) Evolution of the non-dimensional critical flow velocity for flutter instability $U^{*}$ as function of the non-dimensional gap $c$ for three different plates. Plate 1: $M^{*}=0.48, h=0.16$, (o), experiment; $(--)$ $3 \mathrm{D}_{c}$ model. Plate $2: M^{*}=0.48, h=0.2$, $(\square)$ experiment; (- -.) $3 \mathrm{D}_{c}$ model. Plate 3: $M^{*}=0.53, h=0.43,(\Delta)$ experiment; $(-) 3 \mathrm{D}_{c}$ model. Thin lines indicate the values given by the $3 \mathrm{D}_{\infty}$ model, while the dotted line indicates the value given by the $2 \mathrm{D}$ model for $M^{*}=0.5$. Inset: $U^{*}-U_{2 D}$ plotted as function of $\mathrm{c} c$ in a log-log scale, showing that it is governed by a power law of the form $c^{0.15}$.

guished. The first two are viscous effects. In this region of the flow, the fluid's viscosity plays an important role and this may have two opposite consequences. First, viscous friction may stabilize the plate and thus increase the critical velocity. Note that viscous induced damping may also have a destabilizing effect but at high values of the mass ratio not explored in the present analysis. ${ }^{8,18}$ Second, due to the viscosity, the effective clearance gap may be smaller than its actual value, thus giving something closer to the 2D case and consequently a lower critical velocity. However, these two opposite effects of the viscosity may be small in practice, because they are effective in the flow direction but not that important in the direction perpendicular to the flow. The 2D-flag case can be seen as a situation where fluid circulation around the flag in a plane perpendicular to the main flow is not possible. This circulation is not precluded by the presence of a boundary layer, hence, its influence should be small in practice. The last effect is a consequence of the reduction of the axial flow velocity in the boundary layer. Indeed, one may consider that locally, the linearization of the pressure perturbation associated to a small perturbation of the flag's deflection can be separated into three contributions, an added mass term scaling as $\rho$, a Coriolis term scaling as $\rho u$, and a rigidity term, scaling as $\rho u^{2}, u$ being the local axial mean flow velocity. ${ }^{19}$ When $u$ locally decreases in the boundary layer, the aerodynamic reaction due to the Coriolis and rigidity terms decreases as well and their destabilizing effect should be lowered. Consequently, the presence of the boundary layer should be stabilizing. This might explain the slight discrepancy between the experimental data and the 
model at $h=0.16$ and $h=0.2$. At $h=0.43$, the influence of the boundary layer may be smaller, because it is relatively smaller than the flag's height in this case.

In conclusion, we have presented experimental results on the effect of the spanwise clearance on the flutter critical velocity of a cantilevered plate in an axial flow. The experimental results show a good agreement with a model derived in a previous paper. ${ }^{13}$ Velocity profiles and boundary layer thickness have been measured, showing that in the experiments, the gap sizes of interest, i.e., sizes small enough for the critical flow velocity to be significantly affected by the presence of the wall are of comparable magnitude as the boundary layer. It was shown that the dominant effect of the boundary layer should be stabilization so that the destabilization observed when $c$ decreases can only be explained by a blockage effect in a purely potential flow model. Natural extensions of this work include the study of the influence of the other walls of the channel and a more detailed study of the boundary layer. For instance, a new set of experiments, consisting in varying the boundary layer thickness while the other parameters are kept constant, could be performed to properly assess the hypothesis of negligible viscous effects.

${ }^{1}$ Y. Aurégan and C. Depollier, "Snoring: Linear stability and in-vitro experiments," J. Sound Vib. 188, 39 (1995).

${ }^{2}$ L. Huang, "Flutter of cantilevered plates in axial flow," J. Fluids Struct. 9, 127 (1995)

${ }^{3}$ C. Q. Guo and M. P. Paidoussis, "Stability of rectangular plates with free side-edges in two-dimensional inviscid channel flow," J. Appl. Mech. 67, 171 (2000).

${ }^{4}$ A. Kornecki, E. H. Dowell, and J. O'Brien, “On the aeroelastic instability of two-dimensional panels in uniform incompressible flow," J. Sound Vib. 47, 163 (1976).
${ }^{5}$ Y. Watanabe, S. Suzuki, M. Sugihara, and Y. Sueoka, “An experimental study of paper flutter,” J. Fluids Struct. 16, 529 (2002).

${ }^{6}$ L. Tang, M. P. Païdoussis, and J. Jiang, "Cantilevered flexible plates in axial flow: Energy transfer and the concept of flutter-mill," J. Sound Vib. 326, 263 (2009).

${ }^{7}$ J. A. Dunnmon, S. C. Stanton, B. P. Mann, and E. H. Dowell, "Power extraction from aeroelastic limit cycle oscillations," J. Fluids Struct. 27, 1182 (2011).

${ }^{8}$ O. Doaré and S. Michelin, "Piezoelectric coupling in energy-harvesting fluttering flexible plates: Linear stability analysis and conversion efficiency," J. Fluids Struct. 27, 1357 (2011).

${ }^{9}$ M. J. Shelley and J. Zhang, "Flapping and bending bodies interacting with fluid flows," Annu. Rev. Fluid Mech. 43, 449 (2011).

${ }^{10}$ C. Eloy, C. Souilliez, and L. Schouveiler, "Flutter of a rectangular plate," J. Fluids Struct. 23, 904 (2007).

${ }^{11}$ C. Eloy, R. Lagrange, C. Souilliez, and L. Schouveiler, "Aeroelastic instability of cantilevered flexible plates in uniform flow," J. Fluid Mech. 611, 97 (2008).

${ }^{12}$ N. Yamaguchi, T. Sekiguchi, K. Yokota, and Y. Tsujimoto, "Flutter limits and behavior of a flexible thin sheet in high-speed flow. II. Experimental results and predicted behaviors for low mass ratios," J. Fluids Eng. 122, 74 (2000).

${ }^{13}$ O. Doaré, M. Sauzade, and C. Eloy, "Flutter of an elastic plate in a channel flow: Confinement and finite-size effects," J. Fluids Struct. 27, 76 (2011).

${ }^{14}$ G. K. Batchelor, An Introduction to Fluid Dynamics, Cambridge Mathematical Library (Cambridge University Press, Cambridge, 1967).

${ }^{15}$ Y. Yadykin, V. Tenetov, and D. Levin, "The flow-induced vibration of a flexible strip hanging vertically in a parallel flow. Part 1 . Temporal aeroelastic instability," J. Fluids Struct. 15, 1167 (2001).

${ }^{16}$ D. M. Tang, H. Yamamoto, and E. H. Dowell, "Flutter and limit cycle oscillations of two-dimensional panels in three-dimensional axial flow," J. Fluids Struct. 17, 225 (2003).

${ }^{17}$ S. Alben and M. J. Shelley, "Flapping states of a flag in an inviscid fluid: Bistability and the transition to chaos," Phys. Rev. Lett. 100, 074301 (2008).

${ }^{18}$ O. Doaré, "Dissipation effect on local and global stability of fluidconveying pipes," J. Sound Vib. 329, 72 (2010).

${ }^{19}$ M. P. Païdoussis, Fluid-structure Interactions: Slender Structures and Axial Flow (Academic, New York, 2003), Vol. 2. 